\title{
Effectiveness of regular physical activity on exercise tolerance and biochemical parameters in high-risk prostate cancer patients during radiotherapy
}

\author{
Katarzyna Hojan', Owidia Ozga-Majchrzak¹, Maciej Górecki', Ewa Leporowska², Piotr Milecki³ \\ ${ }^{1}$ Department of Rehabilitation, Greater Poland Cancer Centre, Poznan, Poland \\ ${ }^{2}$ Central Laboratory, Greater Poland Cancer Centre, Poznan, Poland \\ ${ }^{3}$ Chair and Department of Electroradiology, Poznan University of Medical Sciences, Poland
}

\begin{abstract}
Introduction. Radiotherapy (RT) is an important modality for curative treatment in high-risk prostate cancer (PCa) patients that improves overall survival, however, it may cause unfavorable changes in physical fitness, increased risk factors for cardiovascular and metabolic complications. The effects of physical exercise on aerobic capacity and on serum levels of liver and renal function indices in PCa patients undergoing RT are still unknown.

Aim. The purpose was to assess the impact of supervised physical activity on aerobic capacity, hemorheology and metabolic biomarker levels in high-risk PCa patients during RT.

Material and methods. Seventy-two men were randomly allocated to two groups before RT for high-risk prostate carcinoma. Thirty-six men conducted physical exercise (EG) and the other 36 men were a control group (UC). Outcomes measured were 6-Minute Walk Test (6MWT), blood parameters, serum levels of hepatic and renal function biomarkers (urea, creatinine, alanine and aspartate aminotransferase, PSA) in a modified shuttle test before and after RT.

Results. After RT, decreased diastolic blood pressure (before test $p=0.05$ ) was observed in the EG and in the UG, there was an increase in resting heart rate $(p=0.017)$, a decrease in walking distance $(p=0.036)$, and an increase in Borg fatigue score during the 6MWT. There was no statistically significant change in renal biomarkers or PSA in the liver in either group.

Conclusions. Physical activity in prostate carcinoma patients during RT improves capacity tolerance with a decrease in Borg fatigue score, but this activity did not influence on serology outcomes or other blood indicators during RT.
\end{abstract}

Keywords: exercises, radiation therapy, oncology, supportive care, rehabilitation.

\section{Introduction}

Radiotherapy (RT) is an important modality for curative treatment in high-risk prostate carcinoma (PCa) patients that improves disease-free and overall survival, especially in locally advanced or higher-risk disease [1]. However, in this group of patients, it may cause unfavorable changes in quality of life, and physical fitness, as well as increases in stress or fatigue [2-5]. Patients with PCa have a high level of interest in life-style changes and are a receptive population for exercise intervention, which, if continued after RT, may have long-term general health benefits in this population of older men [6] Additionally, androgen deprivation therapy (ADT) leads to a number of adverse effects, including deterioration of the musculoskeletal system and increased risk factors for cardiovascular and metabolic complications (e.g. negative lipoprotein profile, abdominal obesity) [7]. 
On the other hand, the important implications of stress generating situations (such as cancer) to changes in blood lipid levels have been widely documented [8]. Apart from its relationship with blood lipid levels, cardiovascular risk has also been related to specific blood parameters $[9,10]$. Increased values of these biochemical parameters are typically associated with liver pathologies. Similarly, urea and creatinine levels are generally considered to be indicators of renal function, although these measures may also be altered in liver and muscle diseases, respectively [11].

The beneficial effect of physical activity on general health and well-being and on mood and mental well-being is well documented and accepted. Exercise training can induce changes in serum concentrations of numerous laboratory parameters [11]. Physical activity can improve cardiovascular efficiency, increase cardiac output and stroke volume, decrease resting heart rate, lower exercise heart rate, and improve ventilation and transport of oxygen from the environment to the cells. Vera and co-authors [12] demonstrate that daily exercise training for a period of one month induces noteworthy modifications of diverse blood biochemical parameters. Exercise training is associated with improved hemorheology and can increase blood volume through an increase in plasma volume and red blood cell mass [13].

It is well documented that physical exercise in patients with localized PCa undergoing RT improved cardiac fitness, flexibility, muscle strength, and overall quality of life, as well as prevented fatigue $[2,3,5]$. The effects of physical exercise on aerobic capacity and on serum levels of liver and renal function in PCa patients undergoing RT are still unknown. Therefore, the aim of this study was an observation of the impact of regular physical exercise on selected blood parameters and endurance tolerance in high-risk PCa patients undergoing RT.

\section{Material and methods}

\section{Setting and Subjects}

This study was conducted between January 2013 and June 2014. The Bioethical Committee at the University of Medical Sciences approved all study procedures (No 10/12). Detailed written and verbal information was provided to participants concerning assessment and training protocols. Participants were informed of the voluntary and confidential nature of the study and were free to withdraw from the study at any time. Informed consent was obtained before participation in the study.

Patients participating in the study met the following eligibility criteria based on a diagnosis of histologically confirmed prostate adenocarcinoma. The definitive RT group had high-risk PCa (T3 or Gleason score $>7$ or PSA $>20 \mathrm{ng} / \mathrm{mL}$ ) and a maximum prostate gland volume of $80 \mathrm{cc}$, age of patients between 18 and 75 years old, scheduled androgen deprivation therapy (ADT) (LHRH analogue every 3 months) planned to continue for a total period of 36 months ( 3 to 5 months prior to RT, during and after completion), no distant metastases, no endocrinological, rheumatic, or cardiac diseases resulting in circulation failure (above Stage II Heart Failure according to the New York Heart Association), no absorption disorders, no other tumors, and good general health (ECOG performance status 0-1).

Exclusion criteria were distant metastases and/or disease progression resulting in RT or the introduction of chemotherapy, insufficiently controlled arterial hypertension, insufficiently controlled metabolic diseases, withdraw from the study before the 3-month period, or death of a patient during the course of the study. For the analysis of the relationship between heart rate (HR) and 6-min walk distance (6MWD), we excluded any subject on medications that could affect $H R$, such as $\beta$-blockers or calcium channel blockers. Eligible participants were identified by their oncologist.

\section{Design}

This study was a two-arm pilot randomized controlled clinical trial. All patients underwent a series of baseline assessments over 2 days, including completion of an on-study form, clinical record form and a 6-minute walk test (6MWT). After completing all baseline assessments, patients were stratified by diagnosis ( $\mathrm{PCa}$ ) and, subsequently, randomized, using a randomization to the control condition consisting of radiotherapy alone or the intervention condition consisting of radiation therapy plus an individually tailored exercise program. Patients randomized to the control group were instructed not to begin any new formal physical exercise programs. This study was not fully blinded; however, the condition allocation was concealed from the patient and physiatrist until after the completion of the baseline assessments. A clinical research coordinator obtained patient consent, collected all the self-reported assessments, and explained the exercise program to participants. The study statistician and data managers remained blinded at all times.

\section{Radiation treatment}

All RT patients received a total dose of 76 Gy in 38 fractions. Prior RT neoadjuvant ADT was started, and then given during the course of RT and thereafter up to 24 months. In the first phase of therapy the pelvic 
lymph nodes with prostate gland and seminal vesicles were included to the dose of 46 Gy at 2 Gy fractions. In the second phase of therapy the irradiated volume was limited to prostate gland plus seminal vesicles to a total dose of 76 Gy. Quality assurance for RT was achieved by daily fiducial marker matching. The clinical target volume included the prostate gland and seminal vesicles but not the pelvic lymph nodes. Patients were positioned in the supine position on the therapeutic machine with a comfortably full bladder and empty rectum $[1,14]$. The time interval between beginning neoadjuvant ADT and RT was more than three months but not longer than five months. For each patient, the following organs at risk with constraint doses were established: rectum, bladder, and femoral heads.

\section{Assessment}

In the classified group of patients, the following schedule was used: Assessment I (Baseline) one week before the onset of RT and ADT and Assessment II, 1 week after the end of RT (after 8 weeks of regular physical exercises).

\section{6 - Minute Walk Test}

Aerobic capacity was estimated using a 6 MWT protocol, which has been used extensively in clinical exercise trials to estimate aerobic capacity in cancer patients $[15,16]$. Recent studies concluded that this method possesses excellent measurement properties, is better tolerated, and is more reflective of activities of daily living than any other walk test in use $[15,17]$.

Participants were given a short warm-up and then asked to walk for a total of 6 minutes, covering as much distance as possible. Two 6MWTs were performed following ATS guidelines [18]. The evaluated parameters were $6 \mathrm{MWD}$ in $\mathrm{m}$ and changes in oxygen saturation (SpO2) and HR during exercise measured via pulse oximetry with a VM-2101-Finger Oximeter - Viamed, United Kingdom. The longest 6MWD of two tests (performed the same day and separated by $20 \mathrm{~min}$ ) was the primary outcome measure. The 6MWT was followed by a short cool-down period and conducted in a hospital corridor, $30 \mathrm{~m}$ long. Patients were instructed to walk the corridor from one end to the other as many times as possible within the permitted time. Upon completion of the test, the total distance walked was recorded and used to estimate aerobic capacity. Secondary measures included fatigue (dyspnea) after test using a modified Borg scale (0-10) [18].
Blood parameters analysis

Venous blood samples were taken to measure prostate-specific antigen (PSA), hemoglobin $(\mathrm{Hb})$, white blood cells, red blood cell neutrophils, lymphocytes, monocytes, platelets, serum lipids (total cholesterol, high- and low-density lipoprotein cholesterol - HDL and LDL), and triglycerides - TG). Serum parameters (urea, creatinine, alanine aminotransferase - ALT and aspartate aminotransferase - AST) were also obtained and processed by a centralized laboratory. Biochemical markers were measured using the Cobas 6000TM clinical chemistry analyzer (Roche, Mannheim, Germany). Hematological indices (complete blood count, hemoglobin) were analyzed in EDTA-blood with the XT-2000i TM (Sysmex Corporation, Kobe, Japan).

\section{Physical exercise}

The intervention group conducted physical exercise 5 days per week. The study exercise program began before RT with aerobic exercises to evaluate the effects of RT and ADT prior to the initiation of exercise. The aerobic activities were completed either alone or in groups and took place at the Rehabilitation Ward in the Greater Poland Cancer Center under the supervision of at least one physiotherapist. The optional exercises included brisk walking, running outside or on a treadmill, and various cycling activities. All activities lasted approximately 50-55 min. The workout consisted of a 5-min warm-up and 40 min of one of the activities, followed by a 10-min relaxation period. The physical activity was moderate, with a maximal heart rate of $65-70 \%$ of the maximum heart rate (220 - age) according to American Cancer Society recommendations [19].

Study organizers verified patients' exercise programs through physical activity notebooks that were checked by a physician in the rehabilitation department once a week.

The control group performed their daily physical activity on their own. Patients in this group were given general recommendations for daily physical exercise [20].

\section{Exercise program adherence}

In the course of the study, some breaks from the exercises were allowed, which were to last no more than two days per 8 weeks. Study organizers verified patients' exercise program through physical activity notebooks that were checked by a physician in the rehabilitation department once a week. 
Diet

PCa patients in our study provided a normal, balanced diet (not restricted), which we observed using Mini Nutritional Assessment (MNA) [21].

\section{Statistical data}

The statistical data was analyzed using STATISTICA software (version 10.0 StatSoft, Poland). The results of anthropometric and 6MWT measurements, as well as blood parameters were analyzed. The quantitative data was described through mean and standard deviation. The Shapiro-Wilk test was used to assess normal distribution compatibility. The differences between the results were described using a two-sided Student's t-test and Wilcoxon's test for connected variables, taking into account the size of the $95 \%$ confidence interval $(\mathrm{Cl})$. The results with $p \leqslant 0.05$ were regarded as statistically significant.

\section{Results}

\section{Participants}

Seventy-three patients were randomly allocated into two groups: 36 men were allocated to the aerobic exercise training group (EG) and the other 37 men comprised the usual care group (UG; one of them was excluded from the study due to his absence during Assessment II). There were no significant differences between the groups at baseline in age or BMI. Demographic data of the participants are summarized in Table 1.

\section{Changes in Objectively Measured Outcomes}

The results from the paired t-tests in Table 2 demonstrated differences of 6MWT parameters between the treated patients. In the exercise group (EG), there was a statistically significant increase in oxygen saturation before the 6MWT and a decrease in diastolic blood pressure (before test $-p=0.05$ )

Table 1. Baseline characteristics of the study groups.

\begin{tabular}{ccccc}
\hline Characteristic & Overall Sample ( $\mathrm{n}=72)$ & $\begin{array}{c}\text { Exercise Training } \\
\text { Group }(\mathrm{n}=36)\end{array}$ & $\begin{array}{c}\text { Usual Care Group } \\
(\mathrm{n}=36)\end{array}$ & $p$ \\
\hline Age [years] (Mean+/-SD) & $66.23+/-4.94$ & $65.7+/-6.2$ & $67.9+/-4.9$ & 0.161 \\
\hline Weight [kg] (Mean+/-SD) & $83.25+/-7.50$ & $83.12+/-8.8$ & $85.43+/-6.7$ & 0.882 \\
\hline BMI [kg/m2] (Mean+/-SD) & $28.69+/-3.4$ & $26.42+/-2.8$ & $29.25+/-3.7$ & 0.386 \\
\hline PSA [ng/mL] (Mean+/-SD) & $4.23+/-2.26$ & $4.08+/-1.57$ & $4.73+/-2.28$ & 0.226 \\
\hline Gleason score(Mean+/-SD) & $6.76+/-1.89$ & $7.02+/-1.20$ & $6.88+/-1.92$ & 0.386 \\
\hline
\end{tabular}

SD, standard deviation; BMI, body mass index; PSA, prostate specific antigen

Table 2. Effects of Physical Exercise on 6MWT Outcome Parameters in Prostate Cancer Patients Receiving Radiotherapy

\begin{tabular}{|c|c|c|c|c|c|c|c|c|c|}
\hline \multirow{2}{*}{\multicolumn{3}{|c|}{ Characteristic }} & \multicolumn{2}{|c|}{ Baseline } & \multicolumn{2}{|c|}{ After treatment } & \multicolumn{3}{|c|}{ Change From Baseline to After Treatment } \\
\hline & & & \multirow{2}{*}{\begin{tabular}{|c|}
$\mathrm{M}$ \\
97.41
\end{tabular}} & \multirow{2}{*}{\begin{tabular}{|l|} 
SD \\
1.05
\end{tabular}} & \multirow{2}{*}{\begin{tabular}{|c|}
$M$ \\
97.96
\end{tabular}} & \multirow{2}{*}{\begin{tabular}{|c|}
$S D$ \\
0.86
\end{tabular}} & \multirow{2}{*}{$\frac{M}{+0.55}$} & \multirow{2}{*}{$\begin{array}{c}95 \% \mathrm{Cl} \\
-0.95 \text { to }-0.15\end{array}$} & \multirow{2}{*}{$\frac{p}{0.008^{*}}$} \\
\hline & hofor GMUNT & EG & & & & & & & \\
\hline $\mathrm{SpO}_{2}$ & before bIVIWI & UC & 97.56 & 1.34 & 97.17 & 1.15 & -0.39 & -0.03 to +0.81 & 0.070 \\
\hline \multirow{2}{*}[\%]{} & \multirow{2}{*}{ after 6MWT } & EG & 97.96 & 1.08 & 97.89 & 1.04 & -0.06 & -0.41 to +0.55 & 0.773 \\
\hline & & UC & 97.30 & 1.60 & 97.26 & 1.83 & -0.04 & -0.61 to +0.70 & 0.892 \\
\hline \multirow{2}{*}{ Systolic BP } & \multirow{2}{*}{ before 6MWT } & EG & 143.13 & 17.77 & 138.27 & 16.10 & +4.86 & -1.11 to +10.83 & 0.106 \\
\hline & & UC & 144.26 & 15.57 & 143.26 & 13.87 & -1.00 & -5.28 to +7.28 & 0.744 \\
\hline \multirow{2}{*}[\mathrm{mmHg}]{} & \multirow{2}{*}{ after $6 \mathrm{MWT}$} & EG & 148.82 & 18.89 & 146.03 & 17.14 & -2.79 & -4.30 to +9.89 & 0.427 \\
\hline & & UC & 145.21 & 19.37 & 148.47 & 20.44 & -3.26 & -9.26 to 2.73 & 0.271 \\
\hline \multirow{4}{*}{$\begin{array}{c}\text { Diastolic BP } \\
{[\mathrm{mmHg}]}\end{array}$} & \multirow{2}{*}{ before 6MWT } & EG & 86.13 & 8.32 & 82.00 & 12.07 & -4.13 & 0.45 to 7.81 & $0.028^{*}$ \\
\hline & & UC & 80.52 & 12.56 & 83.91 & 10.07 & -3.39 & -9.15 to +2.37 & 0.235 \\
\hline & \multirow{2}{*}{ after 6MWT } & EG & 85.72 & 10.23 & 83.86 & 9.31 & -1.86 & -2.47 to +6.20 & 0.386 \\
\hline & & UC & 78.95 & 11.25 & 83.78 & 13.71 & +4.82 & -9.64 to -0.00 & $0.049 *$ \\
\hline \multirow{4}{*}{ Pulse Rate [bpm] } & \multirow{2}{*}{ before 6MWT } & EG & 77.72 & 10.97 & 78.10 & 11.57 & +0.37 & -4.34 to 3.58 & 0.845 \\
\hline & & UC & 75.17 & 11.40 & 80.13 & 9.98 & +4.95 & -8.94 to -0.96 & $0.017^{*}$ \\
\hline & \multirow{2}{*}{ after 6MWT } & EG & 89.10 & 13.32 & 90.13 & 12.85 & +1.03 & -5.47 to 3.40 & 0.636 \\
\hline & & UC & 91.82 & 14.81 & 96.04 & 16.14 & +4.21 & -10.83 to 2.39 & 0.199 \\
\hline \multirow{2}{*}{ Distance [m] } & \multirow{2}{*}{ after 6MWT } & EG & 411.65 & 69.95 & 441.41 & 68.38 & +29.75 & -43.46 to -16.05 & $0.000^{*}$ \\
\hline & & UC & 445.56 & 79.17 & 421.95 & 67.54 & -23.60 & 1.60 to 45.60 & $0.036^{*}$ \\
\hline \multirow{2}{*}{ Borg fatigue score } & \multirow{2}{*}{ after 6MWT } & EG & 2.51 & 1.59 & 2.24 & 0.91 & -0.27 & -0.23 to 0.78 & 0.354 \\
\hline & & UC & 2.28 & 0.70 & 2.82 & 1.23 & +0.54 & -0.24 to 1.12 & 0.030 * \\
\hline
\end{tabular}

Abbreviations: M, mean; SD, standard deviation; UC, Usual Care Group; EG, Physical Exercise Group; $\mathrm{SpO}_{2}$, Oxygen Saturation; BP, Blood Pressure 
after RT. The usual care group (UG) demonstrated a statistically significant increase in HR (before RT), and Borg dyspnea score (above 0.5 point) and a decrease 6MWD $(p=0.05)$ after radiation treatment.
After RT in both patient groups, a decrease in most blood parameters was observed without anisocytosis (there was a statistically significant increase in the $E G)$. The liver parameters did not change $(p=0.05)$ in either of the study groups. There was no statistically

Table 3. Effects of Physical Exercise on Serology Outcome Indicators in Prostate Cancer Patients Receiving Radiotherapy

\begin{tabular}{|c|c|c|c|c|c|c|c|c|}
\hline \multicolumn{2}{|c|}{ Characteristic } & \multicolumn{2}{|c|}{ Baseline } & \multicolumn{2}{|c|}{ After treatment } & \multicolumn{3}{|c|}{ Change From Baseline to After Treatment } \\
\hline & & M & SD & M & SD & M & $95 \% \mathrm{Cl}$ & $p$ \\
\hline \multirow{2}{*}{ Hemoglobin [g/dl] } & EG & 8.82 & 0.76 & 8.50 & 0.62 & -0.31 & 0.11 to 0.52 & $0.003^{*}$ \\
\hline & UC & 8.79 & 0.86 & 8.38 & 0.73 & -0.41 & 0.17 to 0.64 & $0.001^{*}$ \\
\hline \multirow{2}{*}{$\begin{array}{l}\text { Red blood cells } \\
{\left[\times 10^{12} / I\right]}\end{array}$} & EG & 4.76 & 0.48 & 4.52 & 0.42 & -0.24 & 0.11 to 0.37 & $0.000^{*}$ \\
\hline & UC & 4.71 & 0.41 & 4.36 & 0.38 & -0.35 & 0.21 to 0.49 & $0.000^{*}$ \\
\hline \multirow{2}{*}{ Hematocrit [l/l] } & EG & 0.42 & 0.03 & 0.39 & 0.02 & -0.02 & 0.01 to 0.03 & $0.000^{*}$ \\
\hline & UC & 0.42 & 0.03 & 0.39 & 0.03 & -0.02 & 0.01 to 0.04 & $0.000^{*}$ \\
\hline \multirow{2}{*}{ Anisocytes } & EG & 12.90 & 0.80 & 13.31 & 1.04 & +0.40 & -0.76 to -0.04 & $0.028^{*}$ \\
\hline & UC & 13.27 & 0.84 & 13.47 & 0.80 & +0.20 & -0.49 to 0.08 & 0.159 \\
\hline \multirow{2}{*}{$\begin{array}{l}\text { White blood cells } \\
\qquad\left[\times 10^{9} / I\right]\end{array}$} & EG & 7.29 & 2.13 & 5.38 & 1.61 & -1.90 & 1.11 to 2.70 & $0.000^{*}$ \\
\hline & UC & 7.36 & 1.64 & 5.57 & 1.11 & -1.78 & 1.26 to 2.21 & $0.000^{*}$ \\
\hline \multirow{2}{*}{ Lymphocytes $\left[\times 10^{9} / I\right]$} & EG & 1.92 & 0.73 & 1.07 & 0.95 & -0.94 & 0.42 to 1.26 & $0.000^{*}$ \\
\hline & UC & 2.26 & 0.66 & 1.13 & 0.44 & -1.10 & 0.89 to 1.31 & $0.000^{*}$ \\
\hline \multirow{2}{*}{ Neutrophils $\left[\times 10^{9} / 1\right]$} & EG & 4.47 & 1.60 & 3.67 & 1.18 & -0.80 & 0.25 to 1.34 & $0.005^{*}$ \\
\hline & UC & 4.15 & 1.26 & 3.47 & 0.87 & -0.68 & 0.28 to 1.07 & $0.001^{*}$ \\
\hline \multirow{2}{*}{ Monocytes $\left[\times 10^{9} / I\right]$} & EG & 0.70 & 0.21 & 0.64 & 0.27 & -0.06 & -0.02 to 0.14 & 0.139 \\
\hline & UC & 0.70 & 0.20 & 0.70 & 0.17 & -0.00 & -0.08 to 0.07 & 0.914 \\
\hline \multirow{2}{*}{ Eosinophils $\left[\times 10^{9} / 1\right]$} & EG & 0.15 & 0.08 & 0.16 & 0.09 & -0.009 & -0.06 to 0.04 & 0.699 \\
\hline & UC & 0.24 & 0.28 & 0.24 & 0.19 & 0.00 & -0.13 to 0.13 & 0.969 \\
\hline \multirow{2}{*}{ Platelets $\left[\times 10^{9} / I\right]$} & EG & 228.00 & 55.71 & 208.30 & 40.20 & -19.69 & 4.48 to 34.90 & $0.013^{*}$ \\
\hline & UC & 230.29 & 39.58 & 211.79 & 42.99 & -18.50 & 4.61 to 32.38 & $0.011^{*}$ \\
\hline
\end{tabular}

Abbreviations: M, mean; SD, standard deviation; UC, Usual Care Group; EG, Physical Exercise Group.

Table 4. Effects of Physical Exercise on Blood Indicators in Prostate Cancer Patients Receiving Radiotherapy

\begin{tabular}{|c|c|c|c|c|c|c|c|c|}
\hline \multicolumn{2}{|l|}{ Characteristic } & \multicolumn{2}{|c|}{ Baseline } & \multicolumn{2}{|c|}{ After treatment } & \multicolumn{3}{|c|}{ Change From Baseline to After Treatment } \\
\hline & & M & SD & M & SD & M & $95 \% \mathrm{Cl}$ & $p$ \\
\hline \multirow{2}{*}{ Urea [mg/dl] } & EG & 38.28 & 9.74 & 33.42 & 9.93 & $-4,85$ & -6.76 to +16.47 & 0.345 \\
\hline & UC & 39.14 & 11.39 & 35.21 & 9.20 & -3.92 & -0.94 to +8.80 & 0.104 \\
\hline \multirow{2}{*}{ Creatinine [mg/dl] } & EG & 0.84 & 0.21 & 0.94 & 0.14 & +0.10 & -0.03 to 0.23 & 0.112 \\
\hline & UC & 1.00 & 0.24 & 0.94 & 0.22 & -0.06 & -0.02 to +0.15 & 0.146 \\
\hline \multirow{2}{*}{ PSA [ng/ml] } & EG & 3.75 & 3.09 & 0.43 & 0.91 & -3.32 & +0.07 to 5.58 & $0.021^{*}$ \\
\hline & UC & 5.51 & 6.45 & 1.61 & 3,09 & -3.89 & +0.81 to +6.96 & $0.016^{*}$ \\
\hline \multirow{2}{*}{ AST [U/I] } & EG & 27.50 & 8.93 & 25.50 & 5.82 & +2.00 & -4.08 to +8.08 & 0.436 \\
\hline & UC & 27.50 & 11.38 & 24.58 & 7.57 & -2.91 & 2.31 to +8.15 & 0.245 \\
\hline \multirow{2}{*}{ ALT [U/I] } & EG & 32.50 & 15.12 & 32.00 & 13.97 & -0.27 & -8.73 to +9.73 & 0.894 \\
\hline & UC & 34.08 & 13.73 & 33.25 & 14.72 & -0.83 & -8.28 to 9.95 & 0.201 \\
\hline \multirow{2}{*}{ Total cholesterol [mg/dl] } & EG & 212.81 & 45.84 & 204.51 & 44.22 & -8.29 & -4.78 to 21.37 & 0.203 \\
\hline & UC & 200.66 & 48.08 & 196.95 & 33.84 & -3.70 & -9.62 to 17.04 & 0.571 \\
\hline \multirow{2}{*}{$\mathrm{HDL}[\mathrm{mg} / \mathrm{dl}]$} & EG & 51.29 & 11.65 & 54.50 & 11.73 & 2.79 & -0.43 to 6.02 & 0.186 \\
\hline & UC & 54.59 & 15.27 & 53.07 & 13.64 & -1.51 & -1.85 to 4.89 & 0.363 \\
\hline \multirow{2}{*}{$\mathrm{LDL}[\mathrm{mg} / \mathrm{dl}]$} & EG & 139.96 & 46.83 & 131.81 & 41.62 & -8.14 & -0.75 to 17.05 & 0.071 \\
\hline & UC & 135.54 & 44.05 & 138.54 & 39.40 & +3.00 & -12.98 to 18.98 & 0.701 \\
\hline \multirow{2}{*}{$\mathrm{TG}[\mathrm{mg} / \mathrm{dl}]$} & EG & 198.61 & 113.71 & 188.50 & 104.19 & -10.11 & -21.10 to 41.33 & 0.510 \\
\hline & UC & 141.33 & 63.68 & 160.66 & 65.54 & +19.33 & -56.99 to 18.32 & 0.299 \\
\hline
\end{tabular}

Abbreviations: M, mean; SD, standard deviation; UC, Usual Care Group; EG, Physical Exercise Group; PSA, prostate specific antigen; ALT, alanine aminotransferase; AST, aspartate aminotransferase; $H D L$, high density lipoprotein; $L D L$, low density lipoprotein; $T G$, triglycerides. 
significant changes in renal parameters (urea and creatinine) after the study either. PSA decreased by a statistically significantly amount in both groups after RT with ADT. In the EG, all measured lipid parameters decreased after RT (total cholesterol, LDL, TG), in contrast to the UG (increase in TG and LDL).

The changes in blood and serum parameters in both PCa patients groups are presented in Table 3 and 4.

\section{Discussion}

Our study suggested that supervised, regular physical activity in high-risk PCa patients during RT improves aerobic capacity, decreases Borg fatigue score after exercise, and corrects lipid parameters. However, the exercise training used in our study did not influence serology outcomes or other blood indicators in patients undergoing RT in either group.

Exercise can contribute to increased physical capacity and can prevent cardiovascular disease in cancer patients $[19,20]$ These findings support previous research by confirming the safety of and benefits stemming from aerobic exercise during RT. The prescribed exercise duration, frequency, and intensity were matched to possible results, as the American Cancer Society recommends for cancer patients [19]. In a study of PCa patients with home-based aerobic training during RT, Windsor et al. [5] observed that patients in the exercise arm of the study appeared to tolerate their treatment better and reported less severe radiation toxicities. After the study, the authors observed an increase in the distance test and in physical functioning. Kapur et al. [4] concluded that a well-defined exercise schedule appears to reduce the severity of rectal toxicity during RT to the prostate. They observed that patients in the exercise arm of the study appeared to tolerate their treatment better and reported less severe radiation toxicities. After our study, we concluded that regular, moderate physical exercise improves capacity tolerance with a substantial decrease in fatigue (dyspnea) after the 6MWT. Similar results have been observed by other authors $[3,5]$.

Ionizing radiation causes a series of hematological alterations, especially profound lymphocytopenia during and after the radiotherapy course [22]. In our study, we observed a significant decrease in hemorheology after RT and study results suggested no significant impact of exercise training on these parameters. Segal et al. [2] did not suggest a relationship between exercise groups in hemoglobin or PSA levels in men receiving $\mathrm{RT}$ for $\mathrm{PCa}$. Our results are comparative to this result. One reason may be that PSA levels in the usual-care group were higher at baseline and had further to decline during the intervention period. In contrast, another study by Drouin et al. [23] suggested that moderate intensity aerobic exercise (of walking for 20 to 45 minutes, 3 to 5 times per week, at $50 \%$ to $70 \%$ of measured maximum heart rates) appeared to maintain erythrocyte levels during RT of breast cancer.

Our data shows that supervised exercise training during RT with ADT improved lipid parameters in PCa patients. These parameters (LDL, TG, total cholesterol) were better when compared with patients without physical activity. Similar observations appeared in two other studies [2, 24]. Our results confirmed the influence of physical activity on lipid profile and its role in cardiovascular protection in PCa patients.

However, this supervised, regular exercise training did not cause significant changes in liver and renal functional biomarkers (urea, creatinine, ALT and AST). Total creatine kinase levels depend on age, muscle mass, and physical activity. High levels of serum creatine in apparently healthy subjects may correlate with physical training status [25]. In our patients, we observed an inverse tendency in both groups, but the changes were not statistically significant, therefore, we can assume that this physical training was not a heavy and long burden for this patient group. The levels of biochemical parameters were normal (good state of health people) before and after RT with ADT.

To our knowledge, the present study is the first to provide information on changes in renal and liver function, as well as blood parameters during physical exercise in men with high-risk PCa treated with RT and ADT. Our positive findings of the benefits stemming from a exercise program during RT must be interpreted cautiously because of several study limitations.

The small size of this study is a limitation that restricts statistical power and may explain why some of the observed changes did not achieve statistical significance.

In summary, the regular physical exercises, in high-risk PCa patients, during RT and ADT improves aerobic capacity tolerance with a decrease in Borg fatigue score after treatment. This moderate intensity activity did not influence most serology outcomes, but improved lipid parameters after RT.

\section{Acknowledgements}

\section{Conflict of interest statement}

The authors declare that there is no conflict of interest in the authorship or publication of contribution. 


\section{Funding sources}

There are no sources of funding to declare.

\section{References}

1. Thompson I, Thrasher JB, Aus G, Burnett AL, Canby-Hagino ED, Cookson MS. et al. AUA Prostate Cancer Clinical Guideline Update Panel. Guideline for the management of clinically localized prostate cancer: Guideline for the management of clinically localized prostate cancer: 2007 update. J Urol. 2007;177(6):2106-31.

2. Segal RJ, Reid RD, Courneya KS, Sigal RJ, Kenny GP, Prud'Homme DG. et al. Randomized controlled trial of resistance or aerobic exercise in men receiving radiation therapy for prostate cancer. J Clin Oncol. 2009;27(3): 344-51.

3. Truong PT, Gaul CA, McDonald RE, Petersen RB, Jones SO, Alexander AS. et al. Prospective evaluation of a 12-week walking exercise program and its effect on fatigue in prostate cancer patients undergoing radical external beam radiotherapy. Am J Clin Oncol. 2011;34(4):350-5.

4. Kapur G, Windsor PM, McCowan C. The effect of aerobic exercise on treatment-related acute toxicity in men receiving radical external beam radiotherapy for localised prostate cancer. Eur J Cancer Care. 2010;19(5):643-7.

5. Windsor PM, Nicol KF, Potter J. A randomised, controlled trial of aerobic exercise for treatment-related fatigue in men receiving radical external beam radiotherapy for localised prostate carcinoma. Cancer. 2004;101(3): 550-557.

6. Bourke L, Gilbert S, Hooper R, Steed LA, Joshi M, Catto JW. et al. Lifestyle changes for improving disease specific quality of life in men on androgen deprivation therapy for advanced prostate cancer. Eur Urol. 2014;65(5): 865-72.

7. Spry NA, Taaffe DR, England PJ, Judge JS, Stephens DA, Peddle-McIntyre $C$, et al. Long-term effects of intermittent androgen suppression therapy on lean and fat mass: a 33-month prospective study. Prostate Cancer Prostatic Dis. 2013;16(1):67-72.

8. Van Doornen LJ, Snieder H, Boomsma DI. Serum lipids and cardiovascular reactivity to stress. Biol Psychol. 1998;47(3):279-97.

9. Islami F, Mańczuk M, Vedanthan R, Vatten L, Polewczyk A, Fuster V. et al. A cross-sectional study of cardiovascular disease and associated factors. Ann Agric Environ Med. 2011;18(2):255-259.

10. Mazza A, Pessina AC, Tikhonoff V, Montemurro D, Casiglia E. Serum creatinine and coronary mortality in the elderly with normal renal function: the CArdiovascular STudy in the Elderly (CASTEL). J Nephrol. 2005;18(5): 606-12.

11. Banfi G, Colombini A, Lombardi G, Lubkowska A. Metabolic markers in sports medicine. Adv Clin Chem. 2012; 56:1-54.

12. Vera FM, Manzaneque JM, Maldonado EF, Carranque GA, Cubero VM, Blanca MJ, Morell M. Biochemical changes after a qigong program: lipids, serum enzymes, urea, and creatinine in healthy subjects. Med Sci Monit. 2007; 13(12):560-6.

13. El-Sayed MS, Ali N, El-Sayed Ali Z. Haemorheology in exercise and training. Sports Med. 2005;35(8):649-70.
14. Huang SH, Catton C, Jezioranski J, Bayley A, Rose S, Rosewall T. The effect of changing technique, dose, and PTV margin on therapeutic ratio during prostate radiotherapy. Int J Radiat Oncol Biol Phys. 2008;71:1057-64.

15. Schmidt K, Vogt L, Thiel C, Jäger E, Banzer W. Validity of the six-minute walk test in cancer patients. Int J Sports Med. 2013;34(7):63-6.

16. Mao J, Zhang J, Zhou S, Das S, Hollis DR, Folz RJ. et al. Updated assessment of the six-minute walk test as predictor of acute radiation-induced pneumonitis. Int J Radiat Oncol Biol Phys. 2007;67(3):759-67.

17. Solway S, Brooks D, Lacasse Y, Thomas S. A qualitative systematic overview of the measurement properties of functional walk tests used in the cardiorespiratory domain. Chest. 2001;119(1):256-70.

18. ATS Committee on Proficiency Standards for Clinical Pulmonary Function Laboratories. ATS statement: guidelines for the six-minute walk test. Am J Respir Crit Care Med. 2002;166(1):111-117.

19. Spence RR, Heesch KC, Brown WJ. Exercise and cancer rehabilitation: A systematic review. Cancer Treat Rev. 2010;36(2):185-94.

20. Rock CL, Doyle C, Demark-Wahnefried W, Meyerhardt J, Courneya KS, Schwartz AL. et al. Nutrition and physical activity guidelines for cancer survivors. CA Cancer J Clin. 2012;62(4):242-74.

21. Vellas B, Guigoz Y, Garry PJ, Nourhashemi F, Bennahum $D$, Lauque S, Albarede JL. The Mini Nutritional Assessment (MNA) and its use in grading the nutritional state of elderly patients. Nutrition. 1999;15(2):116-122.

22. Lissoni $P$, Meregalli $S$, Bonetto $E$, Mancuso M, Brivio $F$, Colciago M, Gardani G. Radiotherapy-induced lymphocytopenia: changes in total lymphocyte count and in lymphocyte subpopulations under pelvic irradiation in gynecologic neoplasms. J Biol Regul Homeost Agents. 2005;19(3-4):153-8.

23. Drouin JS, Young TJ, Beeler J, Byrne K, Birk TJ, Hryniuk WM, Hryniuk LE. Random control clinical trial on the effects of aerobic exercise training on erythrocyte levels during radiation treatment for breast cancer. Cancer. 2006;107(10):2490-5.

24. Galvão DA, Spry N, Taaffe DR, Denham J, Joseph D, Lamb DS. et al. A randomized controlled trial of an exercise intervention targeting cardiovascular and metabolic risk factors for prostate cancer patients from the RADAR trial. BMC Cancer. 2009;9:419.

25. Brancaccio P, Maffulli N, Limongelli FM. Creatine kinase monitoring in sport medicine. Br Med Bull. 2007;8182:209-30.

Acceptance for editing: 2015-04-29 Acceptance for publication: 2015-05-28

Correspondence address: Katarzyna Hojan MD, PhD Department of Rehabilitation The Greater Poland Cancer Center 15 Garbary St, 61-866 Poznan, Poland phone: +48 6188507 05, fax: +48618521948 e-mail: khojan@op.pl 